\title{
Observations of Energetic Ions and Electrons in the Distant Heliosphere: 2001 - 2005.0
}

\author{
Frank B. McDonald ${ }^{1}$, Edward C. Stone ${ }^{2}$, Leonard F. Burlaga ${ }^{3}$, Alan C. \\ Cummings ${ }^{2}$, Bryant C. Heikkila ${ }^{3}$, Nand $\mathrm{Lal}^{3}$, Norman F. Ness ${ }^{4}$, John D. \\ Richardson $^{5}$, and William R. Webber ${ }^{6}$
}
${ }^{1}$ Institute for Physical Science and Technology, University of Maryland, College Park, MD, USA
${ }^{2}$ California Institute of Technology, Pasadena, CA, USA
${ }^{3}$ NASA / Goddard Space Flight Center, Greenbelt, MD, USA
${ }^{4}$ Bartol Research Institute, University of Delaware, Newark, DE, USA
${ }^{5}$ Center for Space Research, Massachusetts Institute of Technology, Cambridge, MA, USA
${ }^{6}$ Department of Physics and Astronomy, New Mexico State University, Las Cruces, NM, USA

\begin{abstract}
As Voyager 1 (V1) moves closer to the heliospheric termination shock (TS), a new energetic particle population is observed: Termination Shock Particle events (TSP). Interplanetary disturbances in the form of merged interaction regions (MIRs) - identified using Voyager 2 (V2) data - have a major effect on the V1 TSP events from their onset to termination along with triggering episodic increases in higher energy ions $(35 \mathrm{MeV} \mathrm{H})$ and $\mathrm{MeV}$ electrons. The nature of these interactions appear to evolve as V1 moves closer to the TS.
\end{abstract}

Keywords: Heliosphere, Termination Shock, Merged Interaction Regions, Solar/Interplanetary Events, Termination Shock Particles

PACS: $96.40 . \mathrm{Cd}, 96.40 . \mathrm{Fg}, 96.40 . \mathrm{Kk}, 96.50 . \mathrm{Bh}, 96.50 . \mathrm{Ci}, 96.50 . \mathrm{Fm}$

\section{INTRODUCTION}

In 2002.54 the energetic particle experiments on $\mathrm{V} 1\left(85 \mathrm{AU}, 34^{\circ} \mathrm{N}\right)$ observed increases in the intensity of $\mathrm{MeV}$ ions and electrons [1,2] that have persisted as highly variable but durable features over most of the ensuing 2.75 years as the spacecraft traveled some $10 \mathrm{AU}$ further from the sun. These increases are characterized by frequent periods of streaming along the expected direction of the interplanetary magnetic field (IPB), relatively flat energy spectra, and a charge composition at low energies resembling that of anomalous cosmic rays as defined by the high $\mathrm{O} / \mathrm{C}$ ratio $[2,3,4]$. The TS remains the most probable source of this new energetic particle population which has been termed Termination Shock Particles (TSP). 
V2 $\left(25^{\circ} \mathrm{S}\right)$ is some $19 \mathrm{AU}$ closer to the sun than V1. Beginning with the Bastille day event (which arrived at V2 in 2000.9 at $62.9 \mathrm{AU}[5,6]$ ), there have been a series of 9 energetic particle increases that persist over some 3-4 solar rotations. These increases can generally be associated with specific periods of solar activity, are accompanied by increases in the solar wind velocity $(V)$ and the IPB, and by moderate decreases in the galactic cosmic ray (GCR) intensity [5,6,7].

Despite a spacecraft separation of some $90 \mathrm{AU}$ and $60^{\circ}$ in heliolatitude there is a close correspondence between the time histories of the first 4 solar/interplanetary (S/IP) events at V2 and V1. After the onset of the TSP events at V1, it is observed that the S/IP transients, identified at V2, have a significant and diverse influence at V1, including large TSP decreases and impulsive increases in high energy protons and 2$15 \mathrm{MeV}$ electrons. In this note we explore the properties of the V2 S/IP events and their effect on the V1 TSPs.

\section{OBSERVATIONS}

This study makes use of the data from the Voyager Cosmic Ray Subsystem (CRS) experiment (E.C. Stone, P.I.) the Magnetic Field (MAG) experiment (N. Ness, P.I.) and the Plasma Subsystem (PLS) experiment (J. Richardson, P.I.).

Of the $9 \mathrm{~V} 2 \mathrm{~S} / \mathrm{IP}$ increases (Fig. 1), 7 are associated with increases in $V$ and IPB and with decreases in the $>70 \mathrm{MeV}$ rate. It is these four-fold indicators of S/IP intensity, $V$ and IPB increases and $>70 \mathrm{MeV}$ GCR decreases that allows the identification of events 5 and 7. Event 6 inversely mirrors the $>70 \mathrm{MeV}$ rate but is different from the other 7 in that it is contained between 2 large MIRs as defined by the increases in $V$ and IPB [8]. The V1/V2 event 2 is well defined but there are no associated IP changes. There are also several increases in $V$ or IPB at V2 that are not associated with changes in the other data sets.

Because of the higher rigidity and velocity of the GCR's, the $>70 \mathrm{MeV}$ rate decreases (Fig. 1d) reflect the properties of the IP disturbances over a greater volume of space that extends beyond the spacecraft. The onset of the GCR decreases and the time of minimum intensity are taken as a measure of the width of the disturbances, MIRs, that are controlling the low-energy S/IP ions. These MIR boundaries are shown as shaded regions in the figures.

Events 1-4 are observed at V1 after a delay of $\sim 0.19$ years. For these events the initial time-history is very similar to that of V2 except the peak intensity is smaller, corresponding to an average radial intensity gradient of $\sim-7 \%$ / AU. However events 1 and 3 at V1 have a unique feature not seen at V2 - a sharp peaked increase some 64 days after the event onset. For event 2 there are several peaked increases followed by two additional short increases between events 2 and 3 .

TSP 1 and 2 start immediately after the predicted passage of MIRs associated with events 4 and 7 . Event 6 , with its flanking IPB and $V$ peaks, is associated with the termination of TSP 1 [8]. The MIR of event 8 produces a rapid decrease in the 2.5 $\mathrm{MeV}$ ion intensity of TSP 2, briefly reducing it to the level of the V2 S/IP event (Fig. 1). Burlaga et al. [9] have shown that the formation of MIR 8 is dominated by the strong, corotating interaction regions observed in the inner solar system. Event 9 at V2 is the product of the Halloween series of large solar events that had occurred in the 


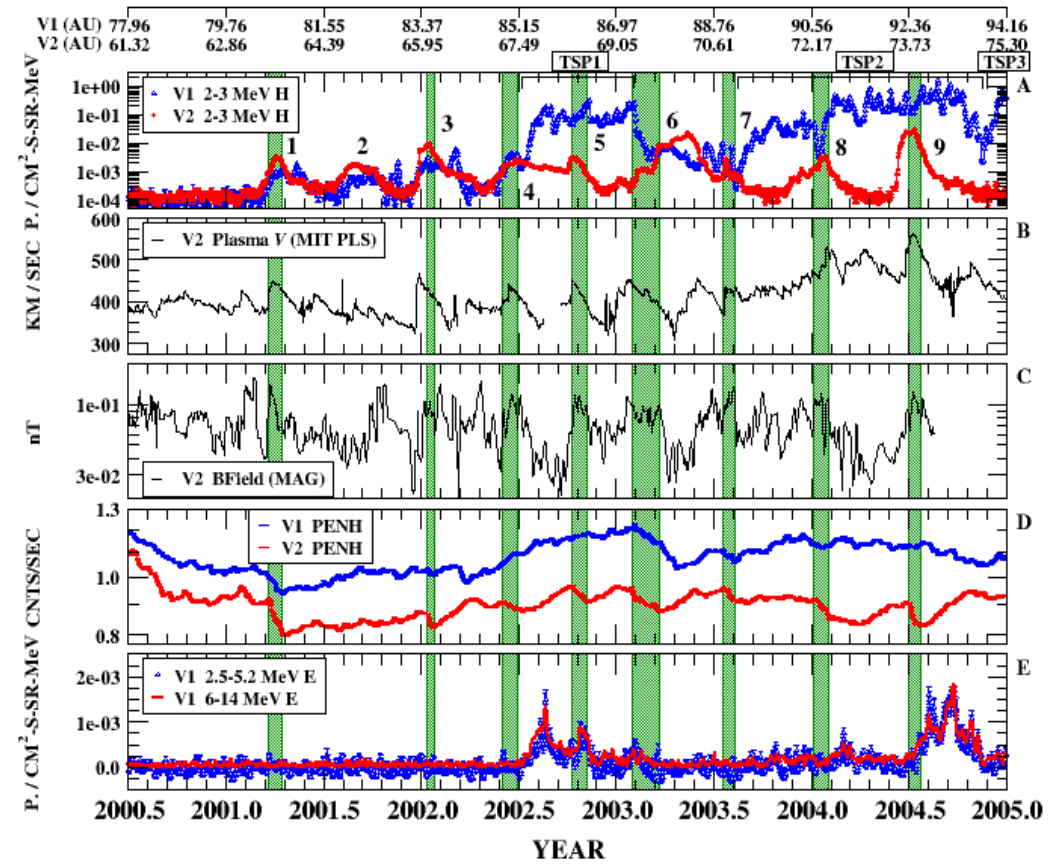

FIGURE 1. A) V1 and V2 $2.5 \mathrm{MeV}$ ion intensity; B) V2 solar wind speed, $V$; C) V2 IPB magnitude; D) V1 and V2 $>70 \mathrm{MeV}$ rate; E) V1 $2.5-5.2$ and 6-14 MeV electrons (Note linear intensity scale for electrons). All of the V2 data in this figure has been time shifted to the location of V1 using a $V$ of 465 $\mathrm{km} / \mathrm{s}$ for the period 2000.1 to 2003.6 and $560 \mathrm{~km} / \mathrm{s}$ for the remainder of the period. The shaded regions are defined by the onset and minimum times of the $\mathrm{V} 2>70 \mathrm{MeV}$ rate. The CRS and PLS data are 5 day moving averages. The MAG data are daily averages.

Sun's southern hemisphere some 6 months earlier, resulting in the largest cycle $23 \mathrm{~S} / \mathrm{IP}$ ion increase yet observed at V2 [10,11].

The background corrected electron intensities from the HET (2.5-5.2 MeV) and TET (6-14 MeV) telescopes are shown in Fig. 1e. There are electron enhancements above the background level associated with the passage of MIRs 5 and 6 and following events 4,8 and 9 . The peak intensities of these two energy intervals are in reasonable agreement, indicating the spectral slope remains essentially constant.

One of the characteristics of the TSP events reported by both the CRS and LECP teams is the frequent observation of strong streaming of energetic ions along the direction of the IPB field $[2,3,4]$ generally in the nominal direction away from the Sun. The look directions of the bi-directional HET telescope are approximately aligned along the nominal $\mathrm{B}$ direction, providing a means of detecting the streaming of 16-56 $\mathrm{MeV} /$ ions [10]. The HET $\mathrm{J}_{\mathrm{A}}$ and $\mathrm{J}_{\mathrm{B}}$ intensities (Fig. 2a) show episodic increases in 35 $\mathrm{MeV} \mathrm{H}$ with the largest following the predicted passage of MIRs 8 and 9. Smaller increases were noted for events 5 and 6 . There are periods when $\mathrm{J}_{\mathrm{B}}$ is much larger than $\mathrm{J}_{\mathrm{A}}$ indicating particle streaming along the field in the direction away from the Sun 


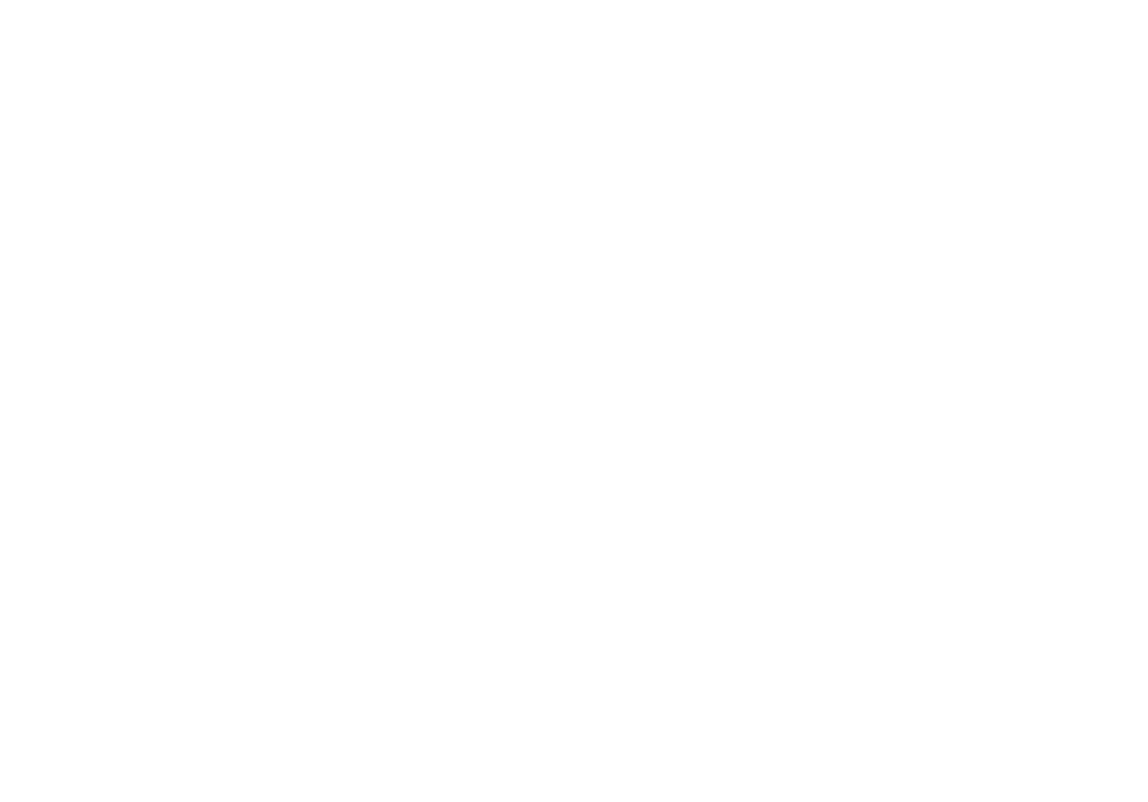

FIGURE 2. A) HET $\mathrm{J}_{\mathrm{A}}, \mathrm{J}_{\mathrm{B}} 30-42 \mathrm{MeV}$ Protons; B) The magnitude of the HET $\mathrm{J}_{\mathrm{A}}$ and $\mathrm{J}_{\mathrm{B}}$ anisotropy. All of the data are 5 day moving averages.

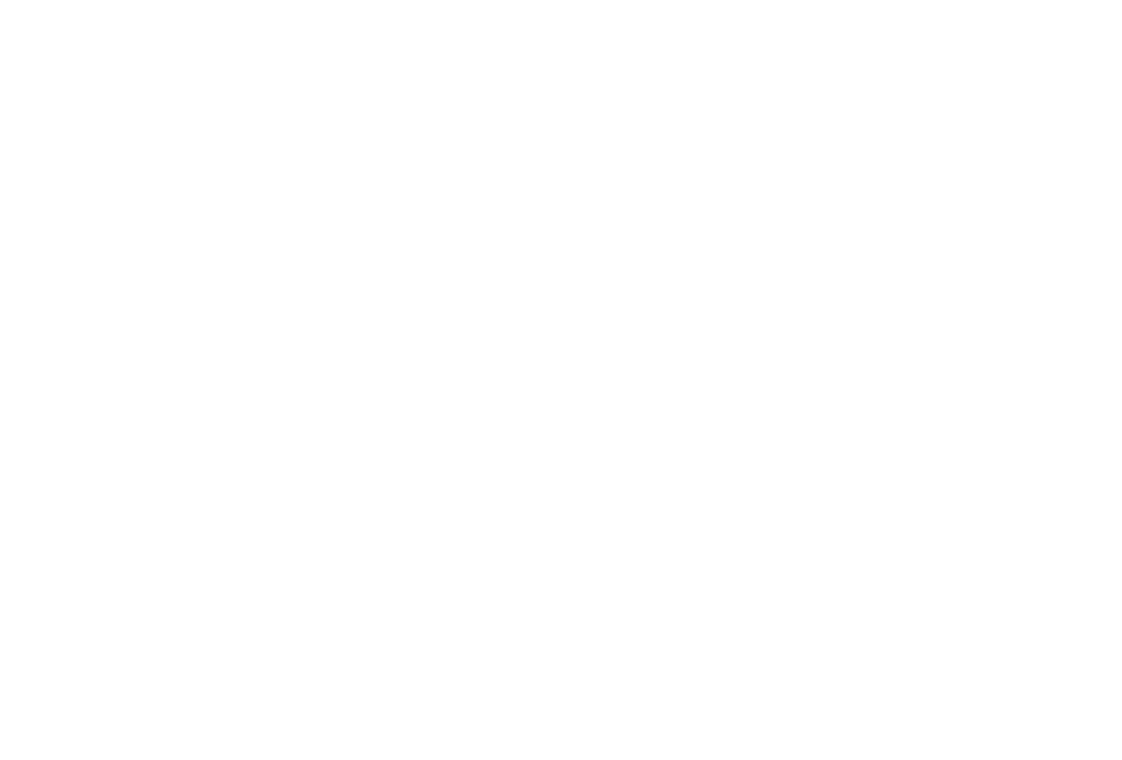

FIGURE 3. A) $2.5-5.2 \mathrm{MeV}$ Electrons; B) $2.5 \mathrm{MeV}$ ion intensity; C) HET $\mathrm{J}_{\mathrm{B}} 30-42 \mathrm{MeV}$ Protons; The $>70 \mathrm{MeV}$ rate is shown in each panel as a solid black line. All of the data are 5 day moving averages. 
consistent with that reported for the lower energy ions. The magnitude of this anisotropy is shown in Fig. $2 b$.

There is a strong correspondence between intensity changes in $35 \mathrm{MeV} \mathrm{H}$ and $\mathrm{MeV}$ electrons as shown in Fig. 3 for the period 2004.0 - 2005.0. Also plotted are the timehistories of $2.5 \mathrm{MeV} \mathrm{H}$ and the $>70 \mathrm{MeV}$ rate is shown in each panel. Short-term fluctuations are assumed to be associated with the presence of local transients in the interplanetary medium. The sharp decrease in $2.5 \mathrm{MeV} \mathrm{H}$ produced by the passage of MIR 8 is followed by an increase in all 4 components (2004.09 - 2004.23) with the temporal structure of the TSPs being similar to that of the GCR.

After the predicted time-of-arrival of the Halloween event at V1 (2004.5) there are large increases and fluctuations in the intensity of the $\mathrm{MeV}$ electrons and the higher energy ions. Again, the fluctuations in all 3 TSP components reflect changes in the GCR rate but now there is a broad decrease in this rate in a series of discrete steps that modulate the TSPs with their minimum intensity coinciding with the GCR minimum, marking the end of TSP 2. This episodic event shows much larger variations, over a longer time period, than the previous ones, possible due to the closer proximity of the TS. The minimum, at 2004.8, is followed by the onset of TSP 3.

\section{DISCUSSION}

As V1 moves closer to the termination shock and with the ongoing recovery toward solar minimum conditions, the lower energy particle observations are dominated by a series of 3 TSP events extending from 2002.54 to 2005.0. This new particle population most probably originates at the TS. The series of S/IP events at V2 have their origin in episodes of solar activity. Thus, V2 is in the solar domain while V1 reflects the activity close to the TS.

At V2 the S/IP events are clearly controlled by MIRs. While the onset of the particle event begins on average some $3 \mathrm{AU}$ before the MIR, the time of the initial peak of the MeV ion intensity coincides with the solar wind peak speed for 7 of the 9 S/IP events. The V1 TSP intensities are generally several orders of magnitude larger than those observed for the V2 S/IP events indicating they have a different source.

At V1 these MIRs play an important role in shaping the time histories of the TSP events. TSP 1 and 2 (Fig. 1) follow immediately after the extrapolated passage of MIRs 4 and 7. The termination of TSP 1 and 2 is related to the passage of MIR 6 and 9.

An unusual feature is the enhancement of $\mathrm{MeV}$ electrons and higher energy ions (Fig. 1, 2, 3) associated with the passage of MIRs 5 and 6 and following events 4, 8 and 9. The electrons are probably of galactic origin that have experienced local reacceleration at the TS [12]. If this is the case then one possible source of the episodic electron and hydrogen ion increases could be further acceleration at the TS by the passage of the MIR.

There is often a difference between the decreases in the GCR rate at V2 and V1 both in form and magnitude. Richardson et al. [13] have examined the relation between the dynamic solar wind pressure at V2 and the V1 TSPs. These dynamic pressure profiles generally define the broad outline of the V2 events and when convected out to $\mathrm{V} 1$, were observed to have a significant role in modulating the V1 
TSPs. Using the $>70 \mathrm{MeV}$ rate provides a sharper definition of the MIR and the modulation effects of MIR 5, 6, 8, and 9 are in agreement with those of Richardson et al. [13].

The preferential streaming of TSPs along the IPB, in a direction away from the Sun, has been interpreted by Cummings and Stone [14] and Jokipii et al. [15] as requiring the shape of the heliosphere to be distorted in such a way that these field lines have encountered the TS prior to reaching V1.

\section{REFERENCES}

1. McDonald, F.B., Cummings, A.C., Stone, E.C., Heikkila, B.C., Lal, N and Webber W.R., Nature 426, 48, 2003

2. Krimigis, S.M., Decker, R.B., Hill, M.E., Armstrong, T.P., Gloeckler, G., Hamilton, D.C., Lanzerotti, L.J. and Roelof, E.C., Nature, 426, 45, 2003

3. Cummings, A.C. et al., Proc. 28 ${ }^{\text {th }}$, Int'l Cosmic Ray Conf. (Tsukuba) 7, 3777, 2003

4. Decker, R.B., Krimigis, S.M., Roelof, E.C. and Hill, M.E., Proc. $28^{\text {th }}$ Int'l Cosmic Ray Conf. (Tsukuba) 7 , 3773, 2003

5. Burlaga, L.F., Ness, N.F., Richardson, J.D. and Lepping, R.P., Solar Physics, 204, 399, 2001

6. Wang, C., Richardson, J.D., Burlaga, L.F., Solar Physics, 204, 411, 2001

7. McDonald, F.B., et al. Physics of the Outer Heliosphere, $3^{\text {rd }}$ Annual IGPP Conf, (ed by V. Florinski) 139, 2004

8. Burlaga, L.F., et al. Journal of Geophys Res., 30, NO 20, 2072, DOI:10.1029/2003 GLO 18 291, 2003

9. Burlaga, L.F., Ness, N.F., Richardson, J.D., McDonald, F.B., and Stone, E.C. Astrophys Journal 618, 1074, 2005

10. Richardson, J.D., Wang, C., Kasper, J.C. and Liu, Y., Geophys Res Letter 32, L03503, DOI:10.1029/2004 GLO20679, 2005

11. Burlaga, L.F., Ness, N.F., Stone, E.C., McDonald, F.B. and Richardson, J.D., Geophys Res Letter 32, L03505, DOI 10.10291/2004 GLO21480, 2005

12. Potgieter, M.S. and Ferreira, S.E.S. Journal of Geophys Res., 107, A7, 10.1029/2001 JA 009040, 2002

13. Richardson, J.D., Journal of Geophys Res., 2005, JA01156R

14. Cummings, A.C. and Stone, E.C., this volume

15. Jokipii, J.R. et al., Astrophys Journal 611, L141, 2004 